\title{
Office-Based Anesthesia
}

\author{
Joseph P. Hunstad, M.D., F.A.C.S. ${ }^{1}$ and Philip H. Walk, M.D. ${ }^{1}$
}

\begin{abstract}
Office-based anesthesia can be safely used to facilitate large, complex plastic surgical procedures. Office-based anesthesia allows for optimal use of the surgeon's time and is cost effective for the patient.
\end{abstract}

KEYWORDS: Safe, complex, cost effective

\begin{abstract}
Ambulatory surgery practiced at freestanding ambulatory surgery centers (ASC) found widespread acceptance beginning in the early 1970s. Plastic surgeons have used this type of facility for extensive procedures since their inception. Many surgeons at the same time found their offices to be convenient places to perform less extensive procedures (e.g., skin lesion removal, blepharoplasty, etc.) as this was cost effective and time efficient for both surgeon and patient. Patients also appreciate privacy afforded to them at the private facility (Fig. 1). Over the past decade, the emergence of officebased anesthesiology (OBA) has allowed extensive surgery to be safely performed in the surgeon's office.

OBA arguably began a century ago when Crawford Long (ca. 1842) administered the first office-based anesthetic. The past decade has seen the first publications of contemporary articles on the subject. Early on, the future of OBA was uncertain because of professional skepticism regarding its safety. Most recently, efforts by the state licensing boards, professional societies (American Society of Anesthesiology, American Society for Aesthetic Plastic Surgery, American Society of Plastic Surgery), and national credentialing bodies (American Association for Accreditation of Ambulatory Surgery Facilities) are overcoming long-standing bias. The status of OBA has been bolstered by attention to relevant clinical and safety issues. Over the past few years, the AAAASF has revamped its standards to address many of the special situations within office-based surgery. ${ }^{1}$
\end{abstract}

\footnotetext{
${ }^{1}$ Hunstad Center for Cosmetic Plastic Surgery, Charlotte, North Carolina.

Address for correspondence and reprint requests: Philip H. Walk, M.D., Chief of Anesthesia Services, Hunstad Center for Cosmetic Plastic Surgery, 8605 Cliff Cameron Drive, Suite 100, Charlotte, NC 28269.
}

\section{ADVANTAGES OF OFFICE-BASED SURGERY FOR LARGE-VOLUME LIPOSUCTION AND BODY-CONTOURING SURGERY}

OBA is defined as the provision of anesthesia services in an operatory that is not licensed as an ASC by the state and that is integrated into the day-to-day operations of a doctor's office. From a patient perspective, office-based surgery (OBS) and OBA involve less paperwork, offer greater privacy, and are less psychologically imposing than hospital-based surgery. Patients should be informed of the accreditation status of the facility (Fig. 2).

One of the reasons surgeons shift procedures to their offices is to increase efficiency. OBS allows one to dovetail consults and follow-up visits with surgical procedures. Also, in many states, ancillary procedures such as laser hair removal require that a physician be on-site when performed. Finally, the cost to the patient is under direct control of the operating surgeon and can be addressed on a case-by-case basis. This is particularly important if touchups or revision surgery is necessary.

If one is to perform complex procedures safely, the physical facility must be adequate. Specific equipment, staff, and environmental factors must be addressed. These standards are addressed by AAAASF and other accrediting institutions. At a minimum, one must have an electrical operating table, appropriate lighting, suction apparatus, and adequate ventilation

Office-Based Plastic Surgery; Guest Editor, Robert A. Ersek, M.D., F.A.C.S.

Semin Plast Surg 2007;21:103-108. Copyright (C) 2007 by Thieme Medical Publishers, Inc., 333 Seventh Avenue, New York, NY 10001, USA. Tel: +1(212) 584-4662.

DOI 10.1055/s-2007-979210. ISSN 1535-2188. 


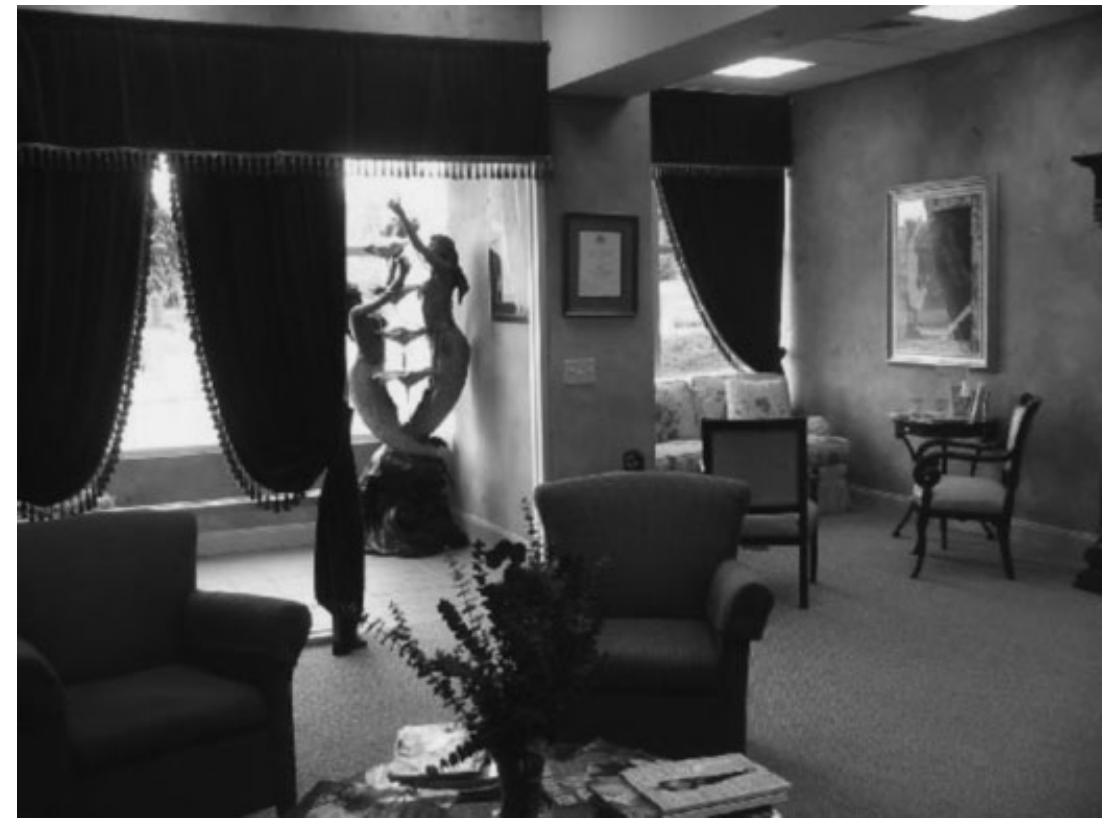

Figure 1 One of the extra benefits of office-based plastic surgery is creating your own "ambiance." Patients then are elevated to a high-quality environment and not subjected to a mass-produced, "low bidder" décor.
(Figs. 3, 4). Personnel who are often cross-trained include nurses and technicians with operating and recovery room expertise. Anesthesia personnel experienced and comfortable working alone in an office is essential.

\section{PATIENT SELECTION}

Proper patient selection depends on sound communication between surgeon and anesthesiologist. Adequate preoperative testing and counseling is dependent on the extent of the planned procedure and preexisting medical condition of the patient. ASA 1, 2 and stable
ASA 3 (patient with well-controlled chronic medical conditions) are candidates for the majority of procedures in our office. Morbidly obese patients, especially those with sleep apnea, are not operated on in the office and are best cared for in a hospital setting. These patients are also counseled to consider bariatric surgery.

Routine preoperative testing is determined by the patient's preexisting medical conditions and the extent of the planned procedure. As an example, if a premenopausal woman has a history of heavy menstrual periods and is undergoing a large-volume liposuction, a hemoglobin level is obtained. The same woman undergoing a breast augmentation with mastopexy would not

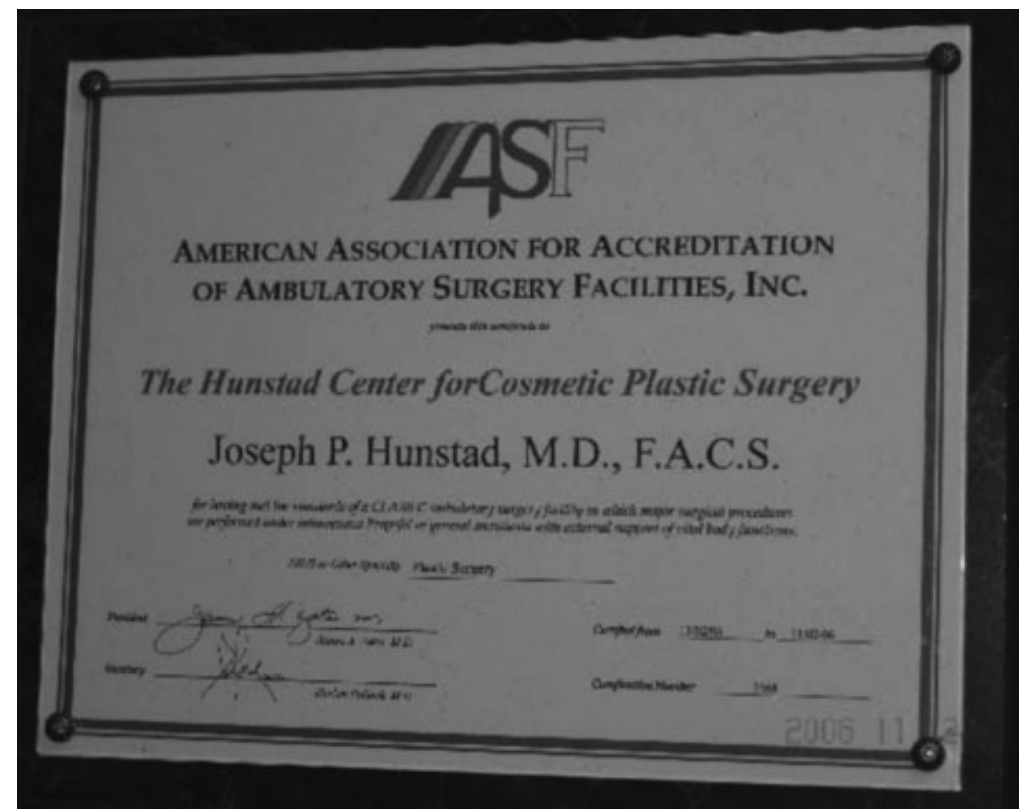

Figure 2 Certification and accreditation builds confidence for the patients and staff. 

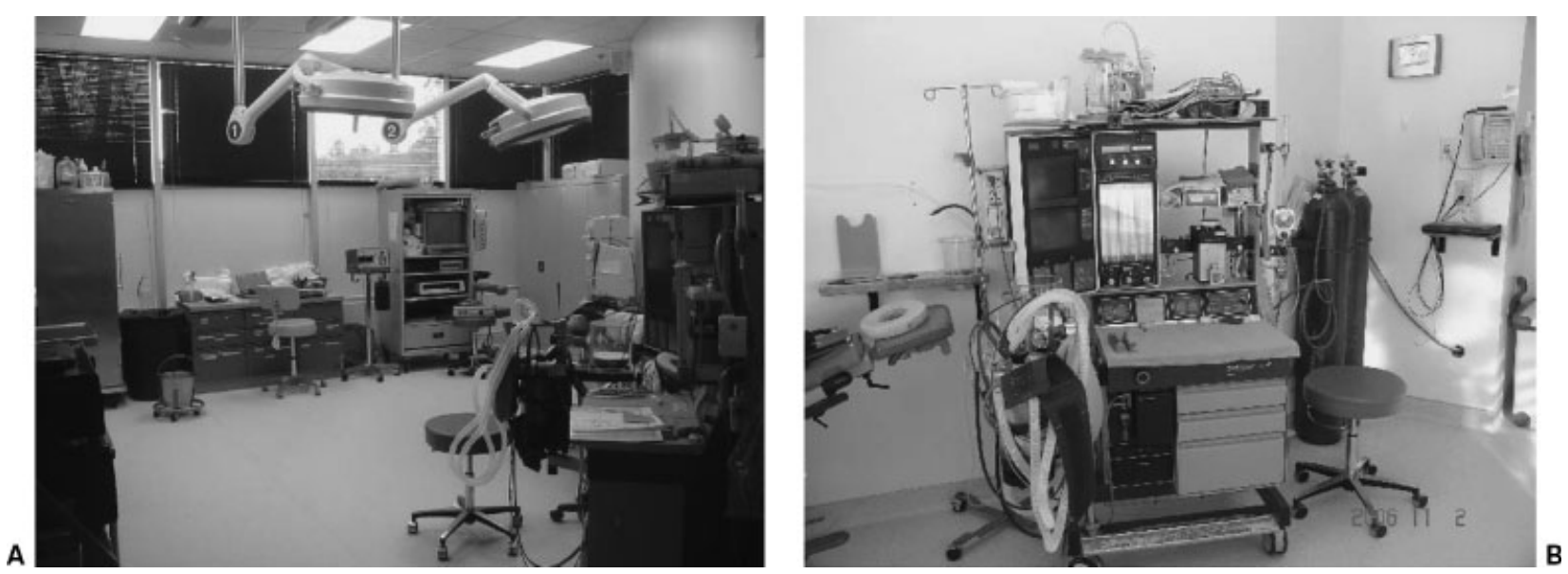

Figure 3 Our facilities are completely equipped for all of our procedures. (A) Overview of operating room. (B) Anesthesia machine.

necessarily warrant such a test. The middle-aged patient over 50 requires special attention regarding cardiac history. If questioning finds appropriate exercise tolerance, a cardiac workup is unnecessary. However, if the patient has episodes of tiredness, shortness of breath, or chest heaviness, be wary and consider medical clearance. Remember that diabetics are susceptible to sudden death without clear-cut evidence of pain of cardiac origin.

\section{ANESTHETIC CHOICE}

Both general anesthesia and monitored anesthesia care (MAC) are being used throughout the country for complex procedures. In our office, we find general anesthesia with control of the airway to provide optimal and safe conditions for these surgeries (Fig. 3A, B). In one study, AAAASF looked at a total of 400,675 procedures over a 5 -year period and found overall mortality specially caused by anesthesia or within 48 hours of surgery totaled five patients. ${ }^{2}$ As regards MAC, especially deep sedation, we recommend that one considers the following observations taken from the ASA Closed Claim Project database of closed malpractice claims. During the 1990s, litigation for injuries arising during MAC became more common. Claims for injuries during MAC represent $1.6 \%$ of claims in the 1970 s, $1.9 \%$ in the $1980 \mathrm{~s}$, and $6.0 \%$ in the 1990s. These trends suggest that an increased risk for litigation from MAC is occurring in spite of the increased use of monitoring such as pulse oximetry. Finally, it is important to note that outcomes of significant severity are occurring with MAC. The data show that there is now a greater proportion of death (34\%) and a higher proportion of brain damage (19\% vs. $12 \%$ for general anesthesia).

Our experience along with studies such as that of the AAAASF allows us to conclude that general anesthesia in the office can be done safely if provided by an experienced provider. In addition, the advantages of safe airway control, optimal operating conditions for the surgeon, and patient comfort are unparalleled when compared with the risk of intraoperative airway emergency and the seesaw effects of intravenous sedation. ${ }^{3}$

\section{ANESTHETIC APPROACH}

The patient for a complex office procedure is counseled both verbally and with written materials before the operation about what to expect. In general, routine medications are continued up to the day of surgery. However, one might consider withholding (2 to 4 weeks) hormonally based birth control medications prior to abdominoplasty and other lower body contouring procedures. These medications increase the risk for deep vein thrombosis and pulmonary embolism. ${ }^{4}$

On arrival at the facility, any remaining questions are answered, and the upcoming procedure is explained to both the patient and caregiver. After preoperative photography and markings are performed, the patient is escorted to the sequential compression device (SCD) and antiembolism stockings are placed on the lower legs prior to the induction of anesthesia. Intravenous access is obtained with a 20 -gauge or larger cannulae (Fig. 4). If procedures involving the arms are planned, an external jugular site should be considered. After intravenous induction and intubation, a Foley catheter with manometer is placed. It is important to secure the intravenous catheter and endotracheal tube carefully as the patient is often turned prone during procedures.

Patient positioning on the operating table is crucial. Soft pads are positioned under the patient's knees and heels and the arm boards are padded and equipped with Velcro ${ }^{\circledR}$ restraints. The vast majority of our patients are tumesced with a lidocaine and epinephrine solution. ${ }^{5}$ The solution is warmed $\left(39^{\circ} \mathrm{C}\right)$ to prevent hypothermia. Intravenous and infiltration solutions are kept constantly warm in a modified food warmer.

The volume and total milligram dose of lidocaine (Abbott Laboratories, Abbott Park, IL) used is based on the surgery being performed and the size of the patient. We use a dilute lidocaine solution of such concentration 


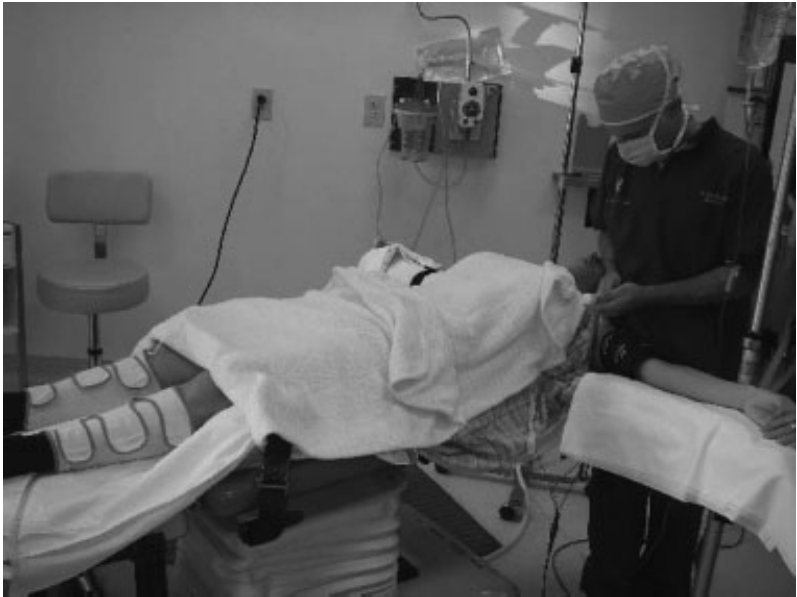

Figure 4 We prefer general anesthesia for most cases.

to allow for a maximum of $35 \mathrm{mg}$ lidocaine per $\mathrm{kg}$ body weight, although higher concentrations have been reported as safe. ${ }^{6}$ On average, we use a volume of 6 to $12 \mathrm{~L}$ of wetting solutions for a large-volume liposuction. We use a chart that we developed in 1992 that shows the mime of tumescent fluid that can be safely infiltrated according to a patient's weight to keep the lidocaine concentration at or below $35 \mathrm{mg} / \mathrm{kg}$ (Fig. 5). After completion of the infiltration portion of the procedure, the body-contouring portion is initiated. As previously stated, this often entails the prone position. We can avoid brachial or lower extremity nerve injury by careful attention to excess extremity movement. The eyes should be carefully taped and padded and appropriate ointment placed prior to turning to avoid corneal abra- sion and compression injury. At the conclusion of the procedure, the patient is awakened and extubated after responding to commands. The patient often assists in moving themselves to the recovery room bed (Fig. 6).

\section{ANESTHETIC MEDICATIONS}

At our center, we anesthetize the patient using a balanced approach that includes intravenous agents and anesthetic gases. Older agents that have cost advantages have proved to be effective in our practice. Newer intravenous agents such as propofol and gases such as sevoflurane are favored by some; however, an outcome advantage cannot be demonstrated. Antiemetics are routinely used and include transdermal scopolamine placed preoperatively, oral dissolving tablet Zofran ${ }^{\circledR}$ (GlaxoSmithKline, London, UK), and intravenous dexamethasone. Pain control is facilitated by liberal use of local anesthetics, pain pumps, and incremental intravenous narcotics.

\section{FLUID REPLACEMENT}

Fluid replacement in these cases includes both the tumescent and intravenous fluids. Our cases find a total aspirate (fat + fluid) of 70 to $90 \%$ or more of the total infiltrate (Fig. 5). Therefore, the ratio of infiltration to aspiration is $\sim 1$ for most major cases. There is additional drainage that occurs during and after surgery that is difficult to account for but can be substantial. The intravenous fluid replacement includes correcting the nil per os (NPO) deficit and residual fluid volume of

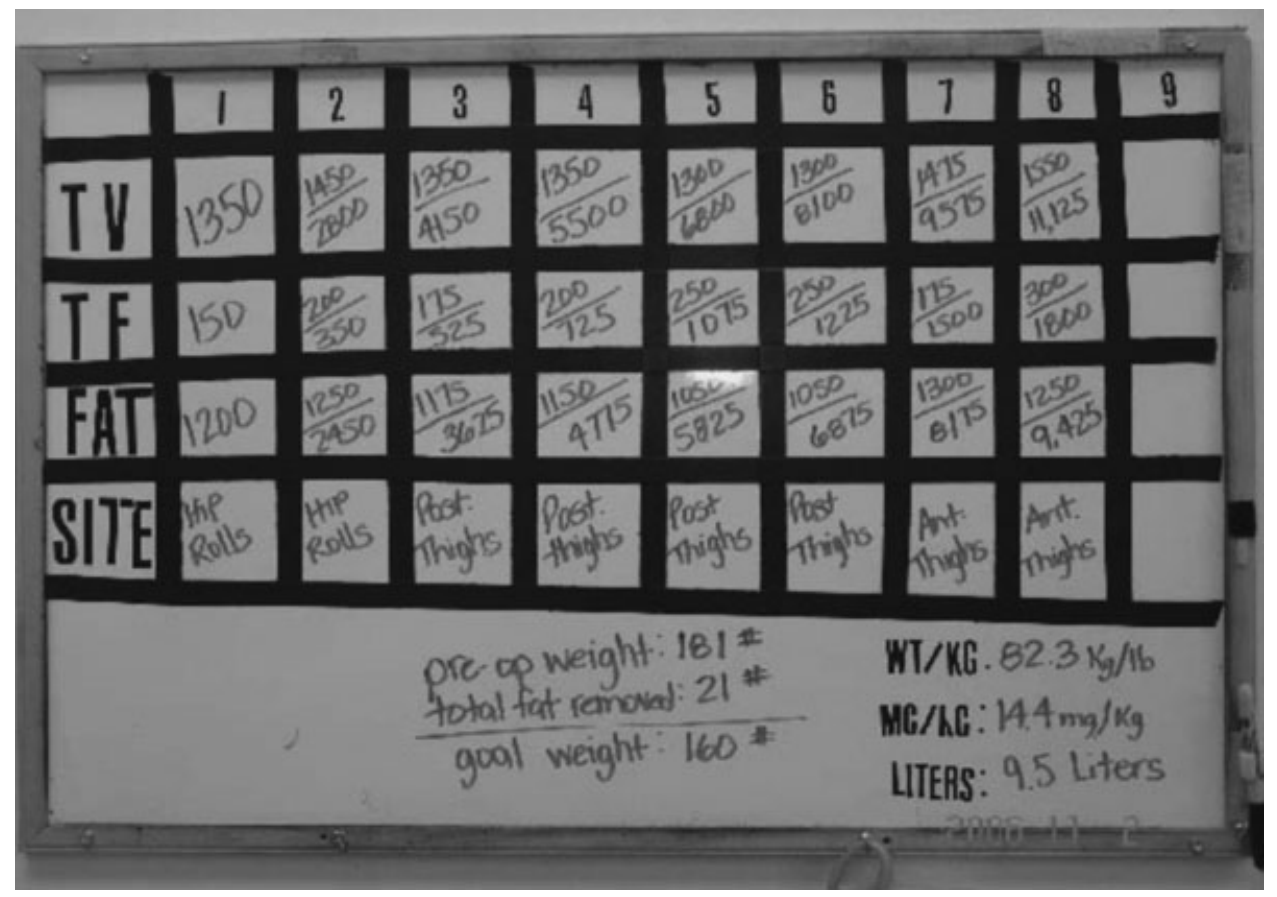

Figure 5 We calculate total volume, total fluid, and total lydocaine doses. 


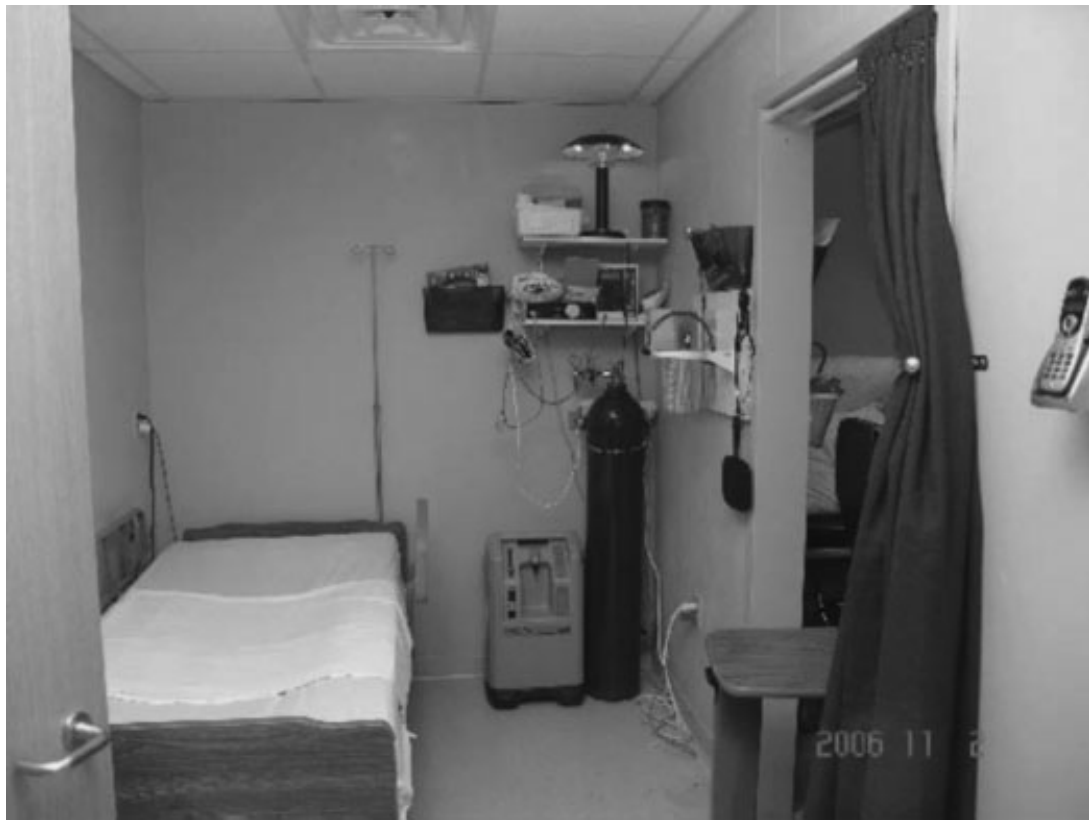

Figure 6 Recovery is private, but our entire staff is just a few steps away.

$90 \mathrm{~mL} / \mathrm{kg} .{ }^{7}$ This provides for adequate fluid replacement and avoids the risk of pulmonary edema. In our practice, a 6- $\mathrm{L}$ replacement of a balanced salt solution is average. This assumes minimal blood loss and a total aspirate as described above.

\section{POSTOPERATIVE CONSIDERATIONS}

Most of our patients remain overnight at our facility, which is equipped with an electrcardiogram (EKG) and pulse oximetry monitors, oxygen source, suction, and an electrical hospital bed (Fig. 3A, B). It is staffed by a registered nurse qualified in advanced cardiac life support (ACLS) and experienced in postoperative care of cosmetic surgery patients. Our immediate concern is for the physiologic stability and comfort of the patient. This is ensured by adequate fluid replacement as assessed by urine output of at least $1 \mathrm{~mL} \mathrm{~kg}^{-1} \mathrm{~h}^{-1}$. Hypothermia is addressed by controlling the temperature of the room during and after the procedure, warming fluids, and by the use of forced-air warming devices. Pain control is as mentioned previously, and supplemental oxygen is provided by mask or cannulae when intravenous narcotics are administered.

Another primary concern is the prevention of deep venous thrombosis. Unfortunately, there is no definitive prevention available outside the use of preoperative vena caval umbrellas. In an attempt to minimize this complication, we have used a three-pronged approach that includes identification of risk factors (e.g., hormonally based birth control), avoidance of dehydration and subsequent hypotension, and early, frequent, and ongoing ambulation. We continue the use of mechanical antiembolism devices in our recovery area and overnight. High-risk patients including those with a previous history of deep venous thrombosis, obesity, or family history of thrombophilic conditions are given LMWH (Lovenox ${ }^{\circledR}$ [Aventis Pharmaceuticals, Bridgewater, $\mathrm{NJ}]$ ) at the time of surgery.

\section{CONCLUSION}

Office-based anesthesia and surgery can provide adequate and advantageous conditions for the performance of complex aesthetic surgical procedures. Our experience over 12 years includes more than 8000 patients undergoing general anesthesia without any major anesthetic complications. Finally, it has provided both surgeon and patient a highly satisfactory professional and cost-effective experience.

\section{REFERENCES}

1. American Association for Accreditation of Ambulatory Surgery Facilities, Inc. Manual for accreditation of ambulatory surgical facilities. Mundelein, IL: AAAASF; 2001

2. Morella DC, Colon GA, Fredericks S, et al. Patient safety and accredited office surgical facilities. Plast Reconstr Surg 1997; 99:1496-1500

3. Marcus JA, Tyrone JW, Few JW, Fine NA, Mustoe TA. Optimization of conscious sedation in plastic surgery. Plast Reconstr Surg 1999;104:1338-1345

4. Anderson FA Jr, Spencer FA. Risk factors for venous thromboembolism. Circulation 2003;107:9-16

5. Hunstad JP. The tumescent technique: an evolution. Lipoplasty Newsletter 1994;11:29

6. Ostad A, Kagegama N, May RL. Tumescent anesthesia with a lidocaine dose at $55 \mathrm{mg} / \mathrm{kgr}$ is safe for liposuction. Dermatol Surg 1997;22:921-927

7. Commons G, Halperin B. Considerations in large-volume liposuction. Semin Plast Surg 2002;16:143-152 